\title{
Overlap Syndrome of Primary Biliary Cirrhosis and Autoimmune Hepatitis with Unusual Initial Presentation as an Acute Hepatic Failure
}

\section{Elleuch Nour*, Ennaifer Rym, Ennaifer Rym, Romdhane Hayfa, Hefaiedh Rania, Cheikh Myriam, Bougassas Wassila, Ben Nejma Houda and Bel Hadj Najet} Departement of gastroenterology, Mongi Slim Hospital in Tunis, Tunisia

${ }^{*}$ Corresponding author: Elleuch Nour, Departement of gastroenterology, Mongi Slim Hospital in Tunis, Tunisia, Tel: +21623341006; E-mail: elleuchghorbel.nour@yahoo.fr

Rec date: Apr 29, 2014, Acc date: June 19, 2014, Pub date: June 27, 2014

Copyright: (c) 2014 Nour E, et al. This is an open-access article distributed under the terms of the Creative Commons Attribution License, which permits unrestricted use, distribution, and reproduction in any medium, provided the original author and source are credited.

\begin{abstract}
Primary Biliary Cirrhosis (PBC) and Autoimmune Hepatitis (AIH) may simultaneously coexist in some patients, designated as PBC-AIH overlap syndrome. Acute hepatic failure is an unusual initial form of presentation of PBCAlH overlap syndrome. We report the case of a 31 year-old woman with autoimmune PBC-AIH overlap syndrome who presented with an acute hepatic failure. She revealed a good response to corticosteroid and ursodeoxycholic acid therapy.
\end{abstract}

Keywords: Primary biliary cirrhosis; Autoimmune hepatitis; Acute hepatic failure; Corticosteroid

\section{Introduction}

Autoimmune Hepatitis (AIH) and Primary Biliary Cirrhosis (PBC) are generally easy to discriminate on the basis of clinical, laboratory, and histological findings. The presence of anti-mitochondrial antibodies seropositivity and cholestatic clinical, laboratory and/or histological features in patients with autoimmune hepatitis indicates the overlap syndrome of autoimmune hepatitis and primary biliary cirrhosis. Severe acute hepatic failure is an unusual initial form of presentation of autoimmune hepatitis and primary biliary cirrhosis overlap syndrome. We present a case of an acute liver failure with overlap features of both PBC and AIH who was successfully treated with corticosteroids without liver transplantation.

\section{Case Report}

A 31-year-old female patient was referred to our Hospital for acute liver failure. One month before admission, she developed poor appetite, fatigue pruritus and jaundice. Blood tests showed serum Alanine Aminotransferase (ALT) 415 IU/l (Normal range: $40 \mathrm{IU} / \mathrm{l}$ ), Aspartate Aminotransferase (AST) $453 \mathrm{IU} / \mathrm{l}$ (Normal range: $40 \mathrm{IU} / \mathrm{l}$ ), Alkaline phosphatase (ALP $145 \mathrm{IU} / \mathrm{l})$ (Normal range: 50 to $130 \mathrm{IU} / \mathrm{l})$, Gamma Glutamyl Transferase (GGT) 53 IU/l (Normal range: 30 IU/l), total bilirubin $206.4 \mathrm{umol} / \mathrm{l}$, and prothrombin time international normalized ratio 2.46. Physical examination on admission revealed jaundice but neither consciousness disturbance nor flapping tremor. The abdomen was tender and distended. Abdominal ultrasonographic examination showed mild ascites and hepatosplenomegaly. Doppler ultrasound color flow imaging showed inversion of venous flow, suggesting portal hypertension and no vein hepatic thrombosis was found. Endoscopic findings did not reveal any esophageal or gastric varices. Serological markers for all hepatotropic viruses such as A, B, C, D, E were negative. No signs of an infection with viruses such as cytomegalovirus, Epstein-Barr virus, herpes simplex virus or human immunodeficiency virus were found. Induced liver injury by drug, toxic, herbal remedies or alcohol was completely ruled out.
Quantitative serum immunoglobins revealed IgG: $24.5 \mathrm{~g} / \mathrm{l}$ (normal range: 8-18 g/l) and IgM: $4.1 \mathrm{~g} / \mathrm{l}(0.6-2.50 \mathrm{~g} / \mathrm{l})$. We detected high levels of antinuclear antibody $1 / 160$ and anti-mitochondrial antibody-M2 (AMA-M2): 1/40, by immunofluorescency confirmed in two times. Because of the positivity for antinuclear antibody and AMA, especially AMA-M2, we suspected her to have autoimmune liver disease such as overlap syndrome with characteristics of both PBC and AIH. However transjugular liver biopsy was not available at our center and percutaneous route was not possible because of hemostatic defect. As other disease etiologies were excluded, and given the acute severe presentation with biochemical and immunoserological features of autoimmune liver disease, corticosteroids were promptly initiated. She was first treated with $1 \mathrm{mg} / \mathrm{kg}$ body weight of equivalent prednisolone daily for 10 days, and then with $60 \mathrm{mg}$ prednisolone daily for approximately 120 days. The patient improved gradually in 4 weeks. The ascites disappeared on ultrasonographic examination. The cytolysis resolved whereas ALP and GGT rose (ALP: 430 IU/l, GGT: $160 \mathrm{IU} / \mathrm{l})$ Prothrombin time became normal. Percutaneous liver biopsy was performed. Histological examination revealed that the architecture of the liver was preserved, indicating no cirrhosis, but noted fibrous expansion of portal triad, portal inflammation consisting of lymphocytes and plasma cells and periportal piecemeal necrosis, whereas no biliary change were objectivised.She also started taking 800 $\mathrm{mg}$ of ursodeoxycholic acid daily. She was released from our hospital 30 days after admission. After her discharge, she was treated daily with $20 \mathrm{mg}$ of prednisolone, azathioprine $100 \mathrm{mg}$ and $800 \mathrm{mg}$ of ursodeoxycholic acid. Her laboratory results in the last visit, in the 4 months of onset of her disease, were as follows: ALAT: 40U/l, ASAT: 38 U/l, ALP: 240 U/l, GGT: 48 U/l INR: 1.2.

\section{Discussion}

We present a case of a severe acute liver failure with overlap features with both $\mathrm{PBC}$ and $\mathrm{AIH}$, successfully treated with corticosteroids without liver transplantation. To our knowledge, this is the third case of overlap syndrome $\mathrm{AIH} / \mathrm{PBC}$ reported in the literature presenting with an inaugural severe acute failure [1,2]. AIH/PBC overlap syndrome is a clinical entity characterized by the occurrence of both conditions at the same time in the same patient [3]. In the 
Citation: Nour E, Rym E, Rym E, Hayfa R, Rania H, Myriam C, Wassila B, Houda BN, Najet BH (2014) Overlap Syndrome of Primary Biliary Cirrhosis and Autoimmune Hepatitis with Unusual Initial Presentation as an Acute Hepatic Failure. J Liver 3: 160. doi: $10.4172 / 2167-0889.1000160$

Page 2 of 2

presenting case, the diagnosis of overlap AIH/PBC was challenging because we did not found histological features of PBC on liver biopsy, however, inflammatory bile duct lesions are not constantly present in liver biopsy and sampling variability must be considered. Moreover, approximately $10 \%$ of patients with $\mathrm{AIH}$ may be also persistently AMA positive without any histological features of PBC [4]. Nevertheless, for this patient, response to corticosteroids with subsequent worsening of indices of cholestasis that responded to UDCA is in favor of the diagnosis of overlap syndrome AIH/PBC. In addition, the Chazouillères score, defined by the presence of at least two of the three recognized biochemical, serological, and histological criteria of each disease $\mathrm{AIH}$ and $\mathrm{PBC}$, supports the diagnosis of overlap [5]. Notwithstanding, $\mathrm{PBC}$ would rarely cause liver failure; it is not a rapidly progressive disease. To our knowledge, no cases of $\mathrm{PBC}$ with an acute hepatic failure or with fulminant hepatic failure were reported in literature [6-8]. In the other hand, AIH can cause an acute liver failure. For inaugural severe forms of $\mathrm{AIH}$, the management of drug therapy is controversial. It is not clear whether the introduction of corticosteroid therapy can induce a benefit and thus obviate the need for liver transplantation. But it is very important for patients with $\mathrm{AIH}$ and acute presentation such as the present case to be treated as soon as possible, particularly when urgent liver transplantation is not available, as in our country $[9,10]$. Ichai and colleagues indicated that there is a point beyond which $\mathrm{AIH}$ cannot be salvaged by corticosteroid therapy, and this point can be defined only by assessing the immediate response to corticosteroid treatment [11-12]. This assessment can be made over a 2 week interval, which is sufficiently short to avoid the infectious complications associated with protracted immunosuppressive therapy $[11,12]$. In our case, prothrombin time recovered 4 weeks after the administration of corticosteroids. A critical unanswered question in relation to corticosteroid use in this context is the optimal dose and route of administration. The putative benefit of higher dose of steroids, with a higher response rate and a reduced need for liver transplantation, has to be balanced with the possibility of a higher risk of sepsis [13]. In the future, strict severity criteria need to be defined for these severe forms of AIH or overlap AIH/PBC in order to produce guidelines for such patients: liver transplantation or corticosteroid therapy. Moreover, we need to perform a dynamic score to calculate potential improvement in liver function in the context of corticosteroid therapy [14].

\section{Conclusion}

Inaugural severe acute hepatitis is an unusual presentation of overlap syndrome of primary biliary cirrhosis and autoimmune hepatitis. By analogy with $\mathrm{AIH}$, and as shown in the present case, corticosteroid may also be effective in acute liver failure of overlap syndrome AIH/PBC, although liver transplantation should always be considered.

\section{References}

1. Kayacetin E, Köklü S, Temuçin T (2004) Overlap syndrome of primary biliary cirrhosis and autoimmune hepatitis with unusual initial presentation as fulminant hepatic failure. Dig Liver Dis 36: 419-422.

2. Toru Wakamatsu, Tatsuo Kanda, Akinobu Tawada, Tatsuo Miyamura, Masanori Takahashi, et al. (2012). Acute liver failure in an antimitochondrial Antibody-Positive-63 year old man. Case Rep Gastroenterol 6: 394-399.

3. Poupon R, Chazouilleres O, Corpechot C, Chrétien Y (2006) Development of autoimmune hepatitis in patients with typical primary biliary cirrhosis. Hepatology 44: 85-90.

4. Trivedi PJ, Hirschfield GM (2012) Review article: overlap syndromes and autoimmune liver disease. Aliment Pharmacol Ther 36: 517-533.

5. Chazouillères $\mathrm{O}$, Wendum $\mathrm{D}$, Serfaty L, Montembault $\mathrm{S}$, Rosmorduc $\mathrm{O}$, et al. (1998) Primary biliary cirrhosis-autoimmune hepatitis overlap syndrome: clinical features and response to therapy. Hepatology 28: 296-301.

6. Flores A, Mayo MJ (2014) Primary biliary cirrhosis in 2014. Curr Opin Gastroenterol 30: 245-252.

7. Boerg KM, Chapman RW, Hirshfield GM, Lohse AW, Manns MP, et al. (2011), International Autoimmune Hepatitis Group Overlap syndromes: the International Autoimmune Hepatitis Group (IAIHG) position statement on a controversial issue. J Hepatol 54: 374-385.

8. European Association for the Study of the Liver (2009) EASL Clinical Practice Guidelines: management of cholestatic liver diseases. J Hepatol 51: 237-267.

9. Kanda T, Yokosuka O, Hirasawa Y, Imazeki F, Nagao K, et al. (2005) Acute-onset autoimmune hepatitis resembling acute hepatitis: a case report and review of reported cases. Hepatogastroenterology 52: 1233-1235.

10. Kanda, Yokosuka O, Hirasawa Y, Imazeki F, Nagao K, et al. (2006) Occurrence of autoimmune hepatitis during the course of primary biliary cirrhosis: report of two cases. Dig Dis Sci 51: 45-46.

11. Ichai, Duclos-Vallée JC, Guettier C, Hamida SB, Antonini T, et al. (2007) Usefulness of corticosteroids for the treatment of severe and fulminant forms of autoimmune hepatitis. Liver Transpl 13: 996-1003.

12. Czaja AJ (2007) Corticosteroids or not in severe acute or fulminant autoimmune hepatitis: therapeutic brinksmanship and the point beyond salvation. Liver Transpl 13: 953-955.

13. Yeoman AD, Westbrook RH, Zen Y, Bernal W, Al-Chalabi T, et al. (2014). Prognosis of acute severe autoimmune hepatitis (AS-AIH): The role of corticosteroids in modifying outcome. J Hepatol. 2014. pii: S0168-8278(14)00362-6.

14. Czaja AJ (2013) Acute and acute severe (fulminant) autoimmune hepatitis. Dig Dis Sci 58: 897-914. 